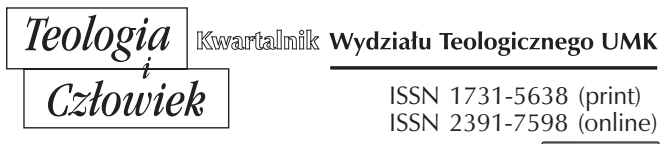

(C) $(\mathbb{\theta} \Theta$

47(2019)3, ss. 33-53

JORDI-AGUSTÍ PIQUÉ I COLLADO OSB

PAPIESKI INSTYTUT LITURGICZNY, RZYM*

PRESIDEPIL@ANSELMIANUM.COM

ORCID: 0000-0002-8487-6581

\title{
ESPERIENZA E/O INTELLIGENZA DELLA FEDE. UN TENTATIVO DI TEOLOGIA-LITURGICA FONDAMENTALE
}

DOI: http://dx.doi.org/10.12775/TiCz.2019.028

Astratto. Vedere e sentire/ascoltare (videre et audire): questi due paradigmi appaiono nel prologo della Dei Verbum (1965). La costituzione dogmatica conciliare ci mette già dall'inizio in questa prospettiva di comprensione della fede e ci propone anche una forma di trasmissione: vedere, ascoltare, soprattutto tramite l'esercizio della comunione.

Nellarticolo si propone di elaborare teologicamente, a partire dalla Dei Verbum ed a partire del «vedere» e «udire» (1 Gv, 1, 2-3), una prospettiva teologica che comprenda

* Nato a Mollerussa nella Catalogna (Spagna); monaco benedettino nell'Abbazia di Montserrat. Insieme al baccalaureato in lettere ha studiato pianoforte all'Escola de Música de Barcelona ed organo con Josep Ma Mas Bonet. Si è laureato in pianoforte ed organo, e in questo ultimo strumento ha ricevuto il Premio di Onore alla fine di carriera; Maestro di Cappella e Direttore dell'Escolania de Montserrat. Ha composto diversi brani musicali per la liturgia e per il coro dell'Escolania di Montserrat. Come organista si è esibito nella Catalogna, Spagna, Francia, Svizzera, Italia, Austria, Germania, Ungheria, Polonia e Korea. Si è dottorato in teologia dogmatica presso l'Università Gregoriana di Roma. Attualmente è professore straordinario e preside del Pontificio Istituto Liturgico di Roma (PIL), vicerettore del Pontificio Ateneo Sant'Anselmo di Roma e professore invitato presso la Facoltà di Teologia di Catalogna (Barcelona) fino a giugno 2019, il Centro Teologico Salesiano Martí-Codolar di Barcellona e della Facoltà Antoni Gaudí di Barcellona; consultore della Congregazione per le Cause dei Santi. 
anche la liturgia per concettualizzare la fede non solo dogmaticamente, ma anche sul piano esperienziale. Ma non dobbiamo dimenticare che se la rivelazione da parte di Dio è chiara ed operante, sperimentiamo una grande difficoltà sul «come parlare di Dio» nelloggi concreto della nostra contemporaneità.

Partendo da questi presupposti si elenca una proposta di teologia-liturgica fondamentale dall'esperienza del vedere e dell'udire (fides ex auditu) come percorso verso l'esperienza di fede che porta a comprensione/intelligenza.

Parole chiave: Teologia e musica; fenomenologia dell'audire; fides ex auditu; esperienza e conoscenza; liturgia; fede; arte; linguaggio.

Summary. Experience and / or Intelligence of Faith. An Attempt at the Theology-liturgical fundamental. Seeing and hearing/listening (videre et audire): these two paradigms appear in the prologue of Dei Verbum (1965). The Council's dogmatic constitution already puts us in the perspective of understanding the faith from the beginning and also proposes a form of transmission: to see, to listen, above all through the exercise of communion.

I propose to elaborate theologically, starting from Dei Verbum and starting from «seeing» and «hearing» (1 Jn 1,2-3), a theological perspective that also includes the liturgy to conceptualize faith not only dogmatically, but also on the experiential plan. But we must not forget that if God's revelation is clear and active, we experience a great difficulty in «how to speak of God» in the concrete today of our contemporaneity.

Starting from these assumptions we list a fundamental theology-liturgical proposal from the experience of seeing and hearing (fides ex auditu) as a path to the experience of faith that leads to understanding.

Key words: liturgy; faith; experience; art; language.

Tre anni fa abbiamo assistito alla Giornata Mondiale della Gioventù celebrata a Cracovia (Polonia) e presieduta da Sua Santità Papa Francesco (dal 25 al 31 agosto 2016). Il mondo ha potuto vedere e udire, tramite la diretta televisiva, la celebrazione della Vigilia di Preghiera e quella dell'Eucaristia. Un momento speciale è stato rappresentato dai canti per l'Eucaristia: il canto del Kyrie e del Gloria sono stati significativamente rilevanti. Eseguiti su testo in latino, hanno proposto una forma musicale contemporanea, dinamica e agile. Si è potuto vedere e udire il senso della Chiesa che si alzava nel mezzo di un grande luogo di culto all'aperto, il cosiddetto "Campus Misericordiae". 
Larte musicale in questa occasione, come quella architettonico a Częstochowa, o quella pittorica nella Capella Sistina in Vaticano, si erge ad autentica icona della fede ${ }^{1}$. Non ne è, però, solo una rappresentazione, è una concrezione formale, viva, eloquente, della medesima fede. L'indifferenza davanti a questa forza performativa dell'arte -malgrado le forme contemporanee e a volte paradossalmente a-formali- non è possibile. Esiste ciò che io oserei chiamare un una compenetrazione meta-dinamica tra arte e fede. La fede, tramite l'arte, si fa vedere e si fa sentire.

Vedere e sentire/ascoltare (videre et audire): questi due paradigmi appaiono nel prologo della Dei Verbum (1965), che adesso prendo come base magisteriale per questa relazione ${ }^{2}$. Citando la prima lettera di San Giovanni: «e vi annunziamo la vita eterna che era presso il Padre e che ci fu manifestata, quel che abbiamo visto e udito, noi lo annunziamo anche a voi, perché voi pure siate in comunione con noi; e la nostra comunione è con il Padre e con il Figlio suo, Gesù Cristo (1 Gv, 1,2-3)» ${ }^{3}$. La costituzione dogmatica conciliare ci mette già dall'inizio in questa prospettiva di comprensione della fede e ci propone anche una forma di trasmissione: vedere, ascoltare, soprattutto tramite l'esercizio della comunione.

Il mio tentativo si propone di elaborare teologicamente, a partire dalla Dei Verbum e a partire del «vedere» e «udire», una prospettiva teologica che comprenda anche la liturgia per concettualizzare la fede non solo dogmaticamente, ma anche sul piano esperienziale. La teologia fondamentale ha fatto questo tentativo - sullesperienza della fede, del fatto di credere e della sua possibilità-, durante l'intero periodo post-

${ }^{1}$ J. Piqué, Teología y música: una contribución dialéctico-trascendental sobre la sacramentalidad de la percepción estética del Misterio. Agustín, Balthasar, Sequeri; Victoria, Schönberg, Messiaen, Tesi Gregoriana, Serie Teología 132, Editrice Pontificia Università Gregoriana, Roma 2006. Idem, «Música Litúrgica: empatía y performatividad», Phase 307 (2012) 74-78. Idem, «Música Sacra/Música Litúrgica: Lenguaje musical y liturgia a cincuenta años de Sacrosanctum Concilium», Phase 317 (2013) 501-515.

2 In un secondo momento citerò testi del Magistero riguardanti la Liturgia e specificamente la musica liturgica.

3 Il Papa Benedetto XVI, nella Lettera apostolica Porta Fidei (2011), chiedeva di tornare ai testi del Vaticano II che i Padri Conciliari ci hanno legato affinché siano conosciuti e assimilati come testi qualificati e normativi del magistero, dentro la Tradizione della Chiesa. Benedetto XVI, «Litterae Apostolicae Motu Proprio datae quibus Annus Fiedi Incohatur» „Porta Fidei”, n. 5, AAS 103 (2011) 725-726. 
conciliare. Dio che rivela se stesso deve essere «visto» e «udito» per essere intelligentemente capito e rendere possibili la trasmissione della fede e una risposta di fede che corrisponda e risponda alla Sua rivelazione. La trasmissione dei contenuti della fede centra la possibilità di dire «Dio» oggi agli uomini e donne del nostro tempo.

Alcuni affermano che la costituzione dogmatica Dei Verbum rappresenta il carattere fondante dell'intero Concilio. Non bisogna dimenticare però che è stata Sacrosanctum Concilium la prima costituzione del Concilio, e proprio i Padri hanno voluto partire della Liturgia per avviare un aggiornamento della fede nel mondo contemporaneo ${ }^{4}$.

Perciò «[...] il centro della vita della Chiesa è il mistero di Dio rivelato in Cristo e comunicato per il Santo Spirito e "così la Chiesa nella sua dottrina, nella sua vita e nel suo culto, perpetua e trasmette a tutte le generazioni tutto ciò che essa è, tutto ciò che essa crede"» (Dei Verbum n. 8$)^{5}$.

Partendo da quest'affermazione, che si trova anche in altre costituzioni conciliari, si può fondare anche una sorta di nuovo orientamento, cioè una "Chiesa ad extra», una Chiesa in costane missione, che stabilisce un dialogo col mondo.

Così dunque, la liturgia diviene incontro col Cristo, che si rende presente personalmente nella sua Chiesa, e allo stesso tempo la Chiesa attende attivamente il suo ritorno in gloria e maestà. Per ciò il rinnovamento liturgico del Vaticano II incoraggia i fedeli a partecipare allazione liturgica, «ma che i fedeli vi prendano parte in modo consapevole, attivo e fruttuoso» (Sacrosanctum Concilium n. 11).

Ma non dobbiamo dimenticare che se la rivelazione da parte di Dio è chiara e operante, sperimentiamo una grande difficoltà relativamente al «come parlare di Dio» nell'oggi concreto della nostra contemporaneità. Dai «preambula fidei», oggi, siamo passati alla «ignorantia fidei» o quantomeno all'ignoranza nella formulazione della fede; dalla ricerca della ragione per comprendere qualcosa del Mistero di Dio, siamo passati all'indifferenza dinanzi a Dio e alla sua realtà: dalla domanda sull' «esistenza

${ }^{4}$ J. Planellas, «Prolusió (4 d’octubre de 2012). Commemoració del cinquantenari de l'inici del Concili Vaticà II», Revista Catalana de Teologia 38 (2013) 344-356.

${ }^{5}$ Ibidem, 347. 
di Dio» siamo passati a una molteplicità di sussidi quasi «idolatrici» che fanno dimenticare la sua presenza viva e operante ${ }^{6}$.

Partendo da questi presupposti elencherò una proposta di teologia-liturgica fondamentale a partire dall'esperienza del vedere e dell'udire come percorso verso l'esperienza di fede che porta a comprensione/ intelligenza. Il primo passo sarà porre in evidenza come la fede ci arriva sempre ex auditu. Il secondo, una visone sulla comprensione dell'arte come «epifania» del Mistero, e proprio come la Parola supera la trasmissione della fede e la sua comprensione. In terzo luogo mi sembra giusto evidenziare alcuni punti magisteriali sullargomento, nello specifico i documenti Sacrosanctum Concilium (1963) e Musicam Sacram (1967) per farne una analisi di attualità. Infine, il punto quarto punto aprirà lo studio alla possibilità della trasmissione della fede e allanalisi dei metalinguaggi come comprensione empatica del Mistero di Dio. Stabiliremo poi alcune conclusioni.

\section{FIDES EX AUDITU: PAROLA, TEOLOGIA, E LINGUAGGl DI TRASCENDENZA}

Il dramma dell'incomunicabilità dell'esperienza di Dio è forse oggi uno dei più gravi problemi della teologia e dell'evangelizzazione. Un problema, però, che deve essere affrontato se si vuole stabilire un dialogo sincero con il pensiero contemporaneo. Forse è vero che la questione dell'esistenza di Dio sia scomparsa dalle nostre aree culturali. Ma dobbiamo ammettere che la questione ora si riformula col paradigma di «come dire Dio?». Penso che il momento attuale, segnato proprio dall'incomunicabilità, sia il momento propizio per poter far scaturire alcune risposte alle sfide del tempo presente che rendano evidente che anche nell'incomunicabilità è possibile pensare e dire Dio. La liturgia è il luogo dove questo problema si fa dialogo di trascendenza, e tante volte questo dialogo passa per il sonoro, per la musica e il canto.

${ }^{6}$ Benedetto XVI, «Litterae Apostolicae Motu Proprio datae quibus Annus Fiedi Incohatur» „Porta Fidei”, n. 10. 
Per Dei Verbum 13, questa domanda trova risposta in Gesù Cristo, Parola definitiva di Dio e culmine della sua Rivelazione. Ci accorgiamo che nell'interpretazione dell'umanità e divinità del Cristo, arte e cultura sono sempre state in dialogo con la formulazione della fede, anche nella più radicale contemporaneità ${ }^{7}$.

La Musica, come arte eminentemente contemporanea e appunto sfuggente, rappresenta un luogo privilegiato di studio ed è un modello ideale per analizzare un processo che si può avviare verso un dialogo tra i nostri contemporanei e l'esperienza estetica.

Visitare teologicamente l'arte contemporanea, e in concreto la musica, è un esercizio da prendere in considerazione quando si vuole proporre una parola su Dio al nostro mondo e agli uomini e le donne di oggi. Credo che l'arte della musica sia tra le espressioni artistiche che più si avvicinano all'esperienza trascendente e, pertanto, meriti attenzione da parte della teologia e anche della cultura ${ }^{8}$. La liturgia è il luogo privilegiato per questa simbiosi.

La sintonia tra teologia -compresa come tentativo di dire una parola rilevante su Dio- e l'espressione artistica -tentativo di dire, con un linguaggio particolare e proprio, una parola su ciò che l'essere umano è- potrebbe stabilirsi come un cammino per scoprire la presenza misterico-simbolica di Dio che si rivela?

E più concretamente, l'eccesso «il plus» di evocazione che la musica possiede, permette di considerarla come un elemento privilegiato per la comprensione esperienziale del Mistero di $\mathrm{Dio}^{10}$. Infatti, lo studio

7 J. Piqué , «L’orecchio pensante. Ascoltare il nome trinitario di Dio. Dal Gregoriano a W.A, Mozart e Ch. Gounod», in La liturgia alla prova del sacro, ed. P. Tomatis, Atti della XXXIX Settimana di Studio dell'Associazione Professori di Liturgia, Convegno Nazionale dei Professori di Liturgia Italia, Brescia 1 settembre 2011, (Studia Liturgica / Nova serie / 57), CLV-Edizioni Liturgiche, Roma 2013, 245-278.

${ }^{8}$ J. Piqué, Teologia e musica. Dialoghi di trascendenza, (Comunicazione/studi), San Paolo, Milano 2013. Idem, "Lattimo fuggente/sfuggente: L'universo sacramentale della musica. Dalla forma estetica all'evento empatico", in Il Corpo del Logos. Pensiero estetico e teologia cristiana, ed. P. Sequeri, Glossa, Milano 2009, 179-195.

9 Cf. E. Salmann, Presenza di Spirito, Messaggero, Padova 2000, 493. Salmann propone la sfida di prendere la cultura, e in concreto anche la musica, come luogo da dove costruire una teologia che permetta formulare il nome di Dio nel momento presente.

${ }^{10}$ H.U. von Balthasar, "Offenbarung und Schönheit», in Verbum Caro: Skizzen zur Theologie, Johannes Verlag, Einsideln 1960, 116. 
della relazione tra teologia e musica come linguaggi di trascendenza e di percezione estetica, non essendo nuovi, sono un capitolo dimenticato del sapere teologico contemporaneo, salvo rare eccezioni ${ }^{11}$.

In Cristo troviamo la chiave del dialogo tra Dio e l'essere umano. Qualsiasi relazione artistica che abbia un nesso tra Parola di Dio, cioè rivelata, e le forme dell'arte, diventa un «apax», un luogo di comprensione empatica che può portare a l'affectus fidei, forse un preludio dell'intelligentia fidei ed anche di Dio che si rivela. Vedere e udire diventano, dunque, elementi dinamici del processo della recezione e del conoscimento della rivelazione. Non ne sono solo preamboli; implicano infatti un certo assenso della fede tramite l'esperienza. Non dimentichiamo che la fede, sempre ci arriva ex auditu: «ex auditu: Ego fides ex auditu auditus autem per verbum Christi» (Rm 10,17).

\section{LA COMPRENSIONE DELL'ARTE COME «EPIFANIA» DEL MISTERO. LA PAROLA, TRASMISSIONE DELLA FEDE E LA SUA COMPRENSIONE}

Dei Verbum (n. 3,4,5), raccogliendo il legato del Concilio di Trento, riprende il tema della Creazione e della profezia come preparazione all'assenso della Fede. Dio si rivela all'essere umano creato da Lui, e gli offre vie di comprensione per arrivare alla grazia che rendono possibile la professione della fede.

Già Melchiorre Cano introdusse, nella sua opera, De Locis Theologicis, stampata nel 1563, il concetto di Locus Theologicus nella teologia cattolica, definendoli come i luoghi da dove il teologo estrae i principia e gli elementi per fondare la sua argomentazione. Cano distinse già tra i sette loci theologici proprii: Bibbia. Tradizione, Chiesa Cattolica, Concili, Magistero dei Papi, i Padri e i Teologi; e d’altra parte i loci theologici alieni, cioè annessi: la ragione naturale, lautorità dei filosofi, il diritto e la storia universale ${ }^{12}$.

${ }^{11}$ J. Piqué, «The Fleeting Moment: The Sacramental Universe of Music, from the Aesthetic Form to the Emphatic Event. Theology and Music: Languages of Transcendence», Res 7 (3/2015) 169-183.

12 Cf. G. Canobbio, Pequeño diccionario de teología, Sígueme, Salamanca 1992, 180. 
Secondo Elmar Salmann, lentamente, nello sviluppo storico, il pensiero teologico e i temi della rivelazione cristiana hanno fatto ingresso nel regno delle arti, dellarchitettura e anche della musica. Lo stesso E. Salmann afferma: «È giunta l'ora di invertire l'ordine, di cogliere la stessa cultura come locus theologicus» ${ }^{13}$. La relazione tra cultura e Chiesa, che in questo momento sembra purtroppo più incline alla rottura, potrebbe trovare una strada di mutuo dialogo e di arricchimento: «Solo in questo modo la rottura tra mistero cristiano e quotidianità, che sembra definitiva, potrà saldarsi (o almeno riconfigurarsi ${ }^{14}$. La teologia contemporanea propone in svariati ambiti una revisione, riconsiderazione e nuova attenzione ai luoghi teologici, mediante un'apertura ai temi che configurano il pensiero contemporaneo e possono essere oggetto di studio teologico. Ho profilato nel mio lavoro unapertura al dialogo -un'apertura dialettica- tra teologia e musica dentro la cornice proposta da Margit Eckholt nella sua Poetik der Kultur $^{15}$ in essa l'autore propone la cultura come nuovo luogo da esplorare per il pensiero teologico contemporaneo. Vorrei proporre una riconsiderazione dei luoghi teologici che ampli gli stessi in direzione della musica, come elemento che porta allesperienza estetica della percezione, quindi da considerare nello studio e nello sviluppo della teologia sistematica.

Per farlo intendo richiamarla proposta già avanzata da Peter Hünermann nel suo Dogmatische Prinzipienlehre, in cui, allargando il concetto dei loci alieni di Cano, l'autore deduce dai documenti del Vaticano II sei nuovi luoghi da proporre alla riflessione dei teologi: le filosofie, il mondo della scienza, la cultura, la comunità, le religioni e la storia ${ }^{16}$. Potremmo igualmente ipotizzare quest'allargamento in conformità a quanto suggerisce Dei Verbum n. 8, che indica nell'esperienza intima spirituale dei credenti il punto di partenza di un eventuale studio teologico. Ci pare chiaro che si possono stabilire linee di connessione tra esperienza estetica ed esperienza spirituale, intendendo entrambe come percorsi di comprensione e percezione della rivelazione del Mistero.

13 E. Salmann, Presenza di Spirito, 485.

${ }^{14}$ Ibidem, 487.

${ }^{15}$ Cf. M. Eckholt, Poetik der Kultur. Bausteine einer interkulturellen dogmatischen Methodenlehre, Herder, Freiburg 2002, 413.

${ }^{16}$ Cf. P. Hünermann, Dogmatische Prinzipienlehre, Aschendorff Verlag, Münster 2003, 223-224; 229-232. 
Da unaltra prospettiva, nellambito del pensiero teologico italiano, Mons. Antonio Staglianò propone la riconsiderazione dei luoghi teologici per arrivare a una comprensione ecclesiologica che recuperi un suo spazio ecclesiale, necessario per la definizione del lavoro teologico e dei suoi compiti: «una teologia come coscienza intellettuale della verità della Chiesa e, nella Chiesa, della fede cristiana ${ }^{17}$. In quest'ambiente la nostra proposta acquisisce una dimensione ecclesiale, poiché il dialogo tra Chiesa e cultura, attraverso una teologia dellarte e, nel nostro caso, della musica, può essere ristabilito e recuperato, così come propone Gaudium et Spes ${ }^{18}$ n. 44.

La teologia può veramente dotarsi di una dimensione emozionale ed empatica, traendola dallattenzione riservata all'arte e, come proponiamo, dalla musica. Tale attenzione le permette di "dire Dio" in maniera sensata ai nostri contemporanei, utilizzando categorie concettuali e verbali affini al nostro tempo e consone a un efficace approccio al senso della rivelazione.

La Bibbia intende la musica come linguaggio per parlare con Dio; non per nulla, contiene al suo interno un libro dedicato al canto nella sua massima espressione e ispirazione: i Salmi. In esso vengono posti in risalto il modo e il tono in cui deve essere cantata l'esperienza del Mistero: «Davanti agli dèi, io canto inni a te» $\left(\right.$ Sal 138,1) ${ }^{19}$.

In concreto, troviamo la ragione fondamentale della musica e del canto nello Psallite sapienter del salmo 47, secondo la traduzione di Gerolamo così amata dalla tradizione benedettina come espressione dell'ideale di vita dei monaci. In questo modo di cantare i salmi, con gusto, assaporandoli sapientemente, si integrano tutti gli elementi dell'essere umano. L'uomo, nella sua interezza, si sente coinvolto nel cantare e nell'essere canto per Dio. Possiamo però parlare di una conformazione dell'uomo a ciò

17 A. Staglianò, Il Mistero del Dio Vivente, EDB, Bologna 1996, 517.

18 «Come è importante per il mondo che esso riconosca la Chiesa quale realtà sociale della storia e suo fermento, così pure la Chiesa non ignora quanto essa abbia ricevuto dalla storia e dall'evoluzione del genere umano. L'esperienza dei secoli passati, il progresso della scienza, i tesori nascosti nelle varie forme di cultura umana, attraverso cui si svela più appieno la natura stessa dell'uomo e si aprono nuove vie verso la verità, tutto ciò è di vantaggio anche per la Chiesa» (GS 44).

19 J. Ratzinger, Cantate al Signore un canto nuovo, Jaca Book, Milano 1996, 120. 
che canta? Se canta, il cantore canta la Parola, e in colui che interpreta il salmo si produce quindi una prima "incarnazione". La musica è, possiamo dire, ermeneuta della Parola.

Il Nuovo Testamento presenta cantici e inni collegati alla grande tradizione dei salmi, come luoghi privilegiati di enunciazione e annuncio teologici. L'esperienza della bellezza non è venuta meno; essa non è stata svelata con l'incarnazione in senso stretto, né tantomeno lo squarcio definitivo del velo del tempio, avvenuto il primo Venerdì Santo, ha lasciato intravedere la grandezza della percezione estetica del Mistero, sebbene lo abbia riempito di contenuto e di significato. La lettura cristologica dei salmi si andrà sviluppando sempre più nel corso dei secoli fino a diventare la chiave esegetica di tutta la Bibbia.

Prima dell'incarnazione del Verbo, e come primo annuncio, Zaccaria canta dopo aver pronunciato il nome di suo figlio Giovanni, e in questo modo esce dal silenzio provocato dalla visione sulla soglia dellantico tempio. Maria canta dopo aver incontrato sua cugina Elisabetta. Simeone canta dopo l'ingresso del Messia nel tempio. Lo stesso coro degli angeli canta per annunciare la meraviglia delle meraviglie: il Verbo si è fatto carne. Cristo stesso cantò con i suoi discepoli inni e cantici rituali. Egli stesso imparò a cantare la berakhâ sul pane e sul vino. La tradizione evangelica vuole che Gesù canti il salmo 21 sulla croce. La Chiesa primitiva riceve tutta questa tradizione ebraica e la fa sua. Gli apostoli salgono al tempio per cantare i salmi di rito. Riuniti con Maria e le donne, nel canto della prima Chiesa, ricevono la visita dello Spirito sotto forma di sonum, dando nuovo respiro alla preghiera e alla predicazione. I cristiani ebrei devono cantare a Dio nei loro cuori.

I Colossesi sono esortati a cantare a Dio con cantici spirituali. Gli inni cristologici, certamente cantati nelle prime celebrazioni liturgiche, erano elaborazioni cristologiche sotto forma di inni, con una pedagogia sapiente ed efficace. Per i neofiti giunti al Cristianesimo senza retroterra ebraico, questi inni, assieme all'interpretazione cristologica dei salmi, saranno la chiave per reinterpretare la Bibbia.

Se la Bibbia inizia con il sonum che aleggiava sulle acque, termina e si conclude con il canto della liturgia celeste. I cantori dell'Apocalisse elevano i loro eterni canti all'Agnello e alla presenza ineffabile del Dio misterioso, indescrivibile a parole. Il testo di Giovanni ci porta ad ascoltare 
gli accordi stessi della liturgia celeste, che purtroppo però ci è concesso di intendere solo attraverso la poesia e la bellezza della composizione letteraria. I canti, le grida, le musiche della moltitudine dei redenti ci giungono soltanto mediante quell'excessus in cui l'autore dell'Apocalisse si vede elevato in una regione definita e descritta da colori e musica; cantano il cantico di Mosè e il grido dell'alleluia: un'inclusione programmatica, per una rilettura totalmente rinnovata.

Così, dunque, la «percezione» della Rivelazione di Dio, si converte nel principale elemento dell'intelligenza della fede. Come diranno i padri della Chiesa, poi, ci sarà bisogno di una catechesi mistagogica che spieghi l'esperienza medesima della fede per arrivarne a formulare «dogmaticamente» i principali enunciati che saranno soggetto di «teologia». L'incontro con Cristo, é il punto culminante di questa esperienza e ne è il fondamento per arrivare alla «possibilità della fede».

\section{ALCUNI PUNTI DEL MAGISTERO: SACROSANCTUM CONCILIUM (1963) - MUSICAM SACRAM (1967)}

Il 4 Dicembre 1963 fu approvato il primo documento del Concilio Vaticano II, la Costituzione sulla Sacra Liturgia Sacrosanctum Concilium, con dieci articoli che si riferiscono alla musica sacra. La musica liturgica è trattata in tutto il capitolo VI.

Larticolo 112, il primo del capitolo VI, rileva che la tradizione musicale di tutta la Chiesa è un patrimonio di grande valore. Si ricorda che le Scritture hanno elogiato il canto sacro, come anche i Padri e i Romani Pontefici, specialmente Pio X, che «ha sottolineato l'aspetto ministeriale della musica sacra nel servizio divino». Una maggiore unione della musica con l'azione liturgica significa maggiore santità e il Concilio, mantenendo i requisiti e le norme di disciplina e della tradizione, osserva che «lo scopo della musica sacra è la gloria di Dio e la santificazione del fedeli» e quindi determina le regole che costituiscono il Capitolo VI (SC 112-121).

Larticolo 114 di SC richiede la conservazione del patrimonio musicale sacro, la creazione delle Scholae Cantorum e dispone che «in ogni azione sacra celebrata in canto, tutta l'assemblea dei fedeli può esercitare la loro partecipazione attiva. Larticolo 116 riconosce il Canto Gregoriano come 
proprio della Chiesa romana e promuove ledizione critica dei libri. L'articolo 117 raccomanda lo sviluppo di un repertorio per le piccole chiese ${ }^{20}$.

Gli orientamenti del Vaticano II in materia di musica liturgica si cristallizzano nello sviluppo dell'Istruzione Musicam Sacram ${ }^{21}$ del 5 Marzo 1967. Tale dichiarazione ha lo scopo di fornire alcune linee guida concrete per l'attuazione delle norme liturgiche emanate dal Concilio e offrire soluzioni ai problemi nel campo della musica liturgica pratica che stanno cominciando ad apparire.

L'Istruzione Musicam Sacram ha subìto un processo accidentato. Le prime applicazioni pratiche della riforma liturgica del Vaticano II hanno messo in allerta i musicisti e i curatori e amanti del canto gregoriano e della polifonia, giacché le chiese cominciarono a essere invase da musica «folk moderna» o «popolare». Le persone coinvolte nella redazione del testo della dichiarazione sono costrette ad accettare le proposte dei vari comitati, insieme ai commentari del Papa Paolo VI stesso, che intervenne direttamente nella formulazione del documento con annotazioni e contributi molto precisi ${ }^{22}$.

L'Istruzione è composta di nove sezioni che riguardano l'attuazione degli orientamenti del Concilio e la sua riforma liturgica a proposito del canto e la musica all'interno della celebrazione liturgica ${ }^{23}$.

Poco dopo arrivarono anche le prime difficoltà nell'attuazione della riforma liturgica e l'Istruzione si proponeva di porvi rimedio. In questo lavoro si evidenziano delle idee che penso siano la chiave per una corretta comprensione dello spirito del documento, per la definizione della funzione della musica:

${ }^{20}$ I successivi articoli elencano altri temi che affermano il ruolo della musica liturgica strumentale (120), lo sviluppo del canto popolare religioso (118), il valore del canto indigeno nei paesi di missione (119), e l'invito agli artisti di incrementare il patrimonio musicale della musica liturgica (121).

${ }^{21}$ Sacra Congregatio Rituum, «Instructio De Musica in Sacra Liturgia. Musicam Sacram», AAS 59 (1967) 300-320.

${ }^{22}$ Cf. A. Bugnini, La riforma liturgica 1948-1975, CLV-Edizioni Liturgiche, Roma 1997, 865-880.

${ }^{23}$ Introduzione, Norme generali, Gli attori della celebrazione, Cantare nella Messa, Ufficio Divino, Lingua, Musica per i testi in lingua volgare, Musica sacra strumentale, e Commissioni di Musica Sacra. 
Lazione liturgica riceve la forma più nobile, se eseguita con il canto, contribuendo ad esso i suoi ministri a secondo il suo grado, e la partecipazione del popolo. Attraverso di essa, la preghiera si esprime in una forma più soave, ed il mistero della sacra liturgia è presentato più chiaramente nella sua propria natura gerarchica e comunitaria, si ottiene l'unione dei cuore più profondamente per l'unità delle voci, le menti più facilmente si elevano alle cose spirituali (sublime), attraverso lo splendore delle cose sacre, e ogni celebrazione prefigura più chiaramente ciò che si svolge nella santa Gerusalemme celeste ${ }^{24}$.

In questa definizione si trovano gli elementi più caratteristici di ciò che caratterizza la musica liturgica, nello spirito della SC. In primo luogo, l'azione liturgica riceve la sua più nobile caratteristica quando eseguita con il canto, ma questo canto è il risultato dell'azione liturgica e personale, sia dei ministri che delle persone coinvolte nell'azione liturgica. Cioè, questa musica non è un mero ornamento, ma è il risultato dello specifico ufficio di ogni singolo nella liturgia. Ma ancora più importante, come il documento fa notare, la preghiera acquista il suo più penetrante e morbido influsso, e manifesta una forma iconica, attraverso l'unione delle voci e il dialogo liturgico, della gerarchia strutturale della liturgia e della comunità. Quest'azione fa elevare tramite la bellezza del sacro, unisce i cuori ed eleva gli spiriti verso l'invisibile: verso la prefigurazione e la percezione della liturgia della Gerusalemme celeste.

Tutto questo processo dimostra la seria attenzione che richiede la musica nello studio della liturgia prima e immediatamente dopo il Vaticano II. Ma la realtà del processo di rinnovamento è stata caratterizzata, in primo luogo, dall'opera di molti artisti e liturgisti; ha visto poi l'irruzione di nuove ondate di espressione musicale che andavano oltre il quadro della riforma liturgica stessa ${ }^{25}$.

24 «Formam nobiliorem actio liturgica accipit, cum in cantu peragitur, ministris cuiusque gradus ministerio suo fungentibus, et populo eam participante. Per hanc enim formam oratio suavis exprimitur, mysterium sacrae Liturgiae eiusque indoles hierarchica et communitatis propria apertius manifestantur, unitas cordium per vocis unitatem profundis attingitur, mentes per rerum sacrarum splendorem ad superna facilius extolluntur, et universa celebratio illam clarius praefigurat, quae in sancta civitate Ierusalem peragitur», Sacra Congregatio Rituum, «De Musica in Sacra Liturgia», 301.

${ }^{25}$ Cf. G. Stefani, «Musica, Chiesa, Società. A 30 anni dal Concilio Vaticano II», 


\section{LA COMPRENSIONE EMPATICA DEL MISTERO DI DIO. LA TRASMISSIONE DELLA FEDE E I «META-LINGUAGGl»}

Dei Verbum chiude il capitolo primo con l'affermazione: "Con la divina La Rivelazione Dio volle manifestare e comunicare se stesso e i decreti eterni della sua volontà riguardo alla salvezza degli uomini, per renderli cioè partecipi di quei beni divini, che trascendono la comprensione della mente umana» (DV n.6) ${ }^{26}$.

L'estetica si è interrogata a più riprese circa la condizione vera e propria della musica come linguaggio. In realtà non vi è un universale della musica. Ci sono specifici brani che si concretizzano in una fase di un compositore, di una cultura, di modi e di visioni particolari e specifiche $^{27}$. Il cristianesimo, almeno nelloccidente, ha segnato l'evoluzione e la comprensione della musica come lingua. In realtà, il cristianesimo ha preso sempre forme musicali, in particolare la parola cantata, per diffondere le sue idee e celebrare la sua liturgia. La semantica della musica si adatterà a una particolare comprensione dell'adeguatezza musica-parola/ musica-Parola. La musica, d’altro canto, è sempre stata associata con il movimento degli «affetti», dell'implicazione sensibile, e per questo motivo è stata considerata dalla filosofia e dalla teologia come «linguaggio ambivalente» ${ }^{28}$.

Ma la musica e la teologia si muovono su un terreno comune d'ineffabilità. Largomento centrale della teologia è l'Ineffabile. La musica è caratterizzata della sua ineffabilità. Esse possono quindi essere considerate come linguaggi della trascendenza che trovano la loro unione nell'ambito dell'esperienza sia sensibile sia estetica dell'ineffabile?

Alcune voci della filosofia sono critiche quando si tratta di definire la musica come un linguaggio. Alcuni autori contemporanei confessano la loro difficoltà di prendere in considerazione apertamente la musica come linguaggio espressivo o referente di qualcosa al di fuori dal suo contenuto

en La Musica e il Sacro. Atti dell'Incontro Internazionale di Studi, ed. B. Brumana G. Giliberti, Firenze 1997, 233-241.

${ }^{26}$ Cf. Concilio Vaticano I, Cost. dogm. sulla fede cattolica Dei Filius, cap. 2: Dz 1786 (3005).

27 E. Fubini, Estetica della musica, Il Mulino, Bologna 2003, 23-24.

${ }^{28}$ Cf. Augustinus, Confessiones, X, 33, 50. 
estetico. Suzanne Langer afferma che la musica, pur essendo un linguaggio artistico singolare, è solo una forma simbolica d'espressione. Sulla musica quest'autore afferma: «la sua vita è l'articolazione ma senza affermare nulla, la sua caratteristica è l'espressività, ma non è l'espressione» ${ }^{29}$. Per Leonard Meyer la musica non ha una funzione referenziale, essa trova cioè il suo compimento in se stessa ${ }^{30}$. Theodor W. Adorno ha sostenuto che la musica può essere espressione o forma, a secondo della funzione sociale che prende in ogni società ${ }^{31}$.

Ma dobbiamo riconoscere che la relazione musica-parola ha dato chiari esempi d'una trascendenza che va oltre la musica stessa, soprattutto in un contesto liturgico. Per citare alcuni esempi possiamo parlare di opere contemporanee di grandi compositori che hanno effettuato questa operazione $^{32}$ : Olivier Messiaen ${ }^{33}$ (Livre du Saint-Sacrement), Arvo Pärt (De Profundis) e Cristoforo Penderecki (Passio secundum Lucam).

In questi tre compositori, la dimensione relazionale e l'ermeneutica della musica rispetto alla Parola sono materializzate nell'uso rilevante di tre elementi formali eminentemente contemporanei: il tempo, la forma e i sensi. Tutti e tre si amalgamano in un linguaggio musicale contemporaneo che fa di loro elementi caratterizzanti per la loro assenza/presenza. Rientrano nell'ambito dell'attenzione al tempo, attenzione alla forma e della speciale attenzione al coinvolgimento dei sensi.

O. Messiaen, A. Pärt e C. Penderecki nella loro musica -ciascuno con uno stile proprio- hanno portato l'attenzione sulla categoria della temporalità decostruendola oppure mediante una rielaborazione tridimensionale. La forma prende in ognuno aspetti minimalisti che evitano strutture formali superate e si situa nella microstruttura di un'architettura

29 S. Langer, Philosophy in a New Key: a study in the symbolism of reason, rite, and art, Mentor Book, The New American Library, New York 1951, 240.

${ }^{30}$ L. Meyer, Emotion and Meaning in Music, University of Chicago Press, Chicago 1956, 35.

${ }^{31}$ Cf. Th. W. Adorno, Philosophie der neuen Musik, Mohr, Tübingen 1949.

32 P. Sequeri, Musica e mistica, Librería Editrice Vaticana, Città del Vaticano, 452.

${ }^{33}$ J. Piqué, «Livre du Saint Sacrement. Eucharistie als Manifestation von Erfahrung und Transzendenz», in Musik des Unsichtbaren. Der Komponist Olivier Messiaen (1908-1992) am Schnittpunkt von Theologie und Musik, Ed. Michaela Christine Hastetter, EOS Verlag, St. Ottilien 2008, 104-125. 
musicale propria dellarte contemporanea. E ancora, il rapporto con i sensi si concentra sull'impressione sonora dell'udito (e la vista in Messiaen) cercando, attraverso risorse timbriche e dinamiche, lempatia sonora, nonostante le difficoltà comprensive del discorso armonico-estetico. Con questi tre elementi, tutti e tre i compositori interpretano la Parola ermeneuticamente, portando alla comprensione empatica che va ben oltre qualsiasi spiegazione, ma sempre con un discorso estetico contemporaneo.

Penso che con questi pochi elementi si possa apprendere il senso metalinguistico della musica come linguaggio perché porta a una comprensione empatica della Parola in musica che va oltre la semplice contemplazione estetica ${ }^{34}$. Wladimir Jankélévitch lo esprimeva come segue: «La musica può creare lo stato di grazia che lungo le pagine piene di metafore poetiche non si può ottenere [...] questo ascolto ci fa sentire, rivelandoli improvvisamente, l'ineffabile, l'invisibile, l'impercettibile» ${ }^{35}$.

E proprio qui che si può chiudere il cerchio ermeneutico: la relazione tra la parola e la sua formulazione artistico-empatica sintetizza la non-possibilità dell'acceso a Dio con l'incontro con la sua rivelazione, la sua stessa parola, Cristo stesso e la tradizione, con la sintesi artistica che trapassa la storia umana facendo comprensibile ciò che è ineffabile: ciò che Dio stesso $\dot{e ̀}^{36}$.

${ }^{34}$ Enrico Fubini lo esprimeva così: «Vi è un'indubbia anche se problematica parentela tra la musica e il linguaggio verbale, che da secoli i filosofi cercano di mettere in luce e si potrebbe forse avanzare l'ipotesi che la musica porta alla luce, mette in evidenza, sottolinea e fa emergere ciò che nel linguaggio è soffocato o rimane allo stato latente», Cf. E. Fubini, Estetica della musica, 32.

35 V. Jankëlëvitch, La Musica e l'ineffabile, Bompiani, Milano 2001, 101-102.

${ }^{36}$ «E il vostro mestiere, la vostra missione; e la vostra arte è proprio quella di carpire dal cielo dello spirito i suoi tesori e rivestirli di parola, di colori, di forme, di accessibilità. E non solo una accessibilità quale può essere quella del maestro di logica, o di matematica, che rende, sì, comprensibili i tesori del mondo inaccessibile alle facoltà conoscitive dei sensi e alla nostra immediata percezione delle cose. Voi avete anche questa prerogativa, nell'atto stesso che rendete accessibile e comprensibile il mondo dello spirito: di conservare a tale mondo la sua ineffabilità, il senso della sua trascendenza, il suo alone di mistero, questa necessità di raggiungerlo nella facilità e nello sforzo allo stesso tempo». Cf. Paolo VI, «Alocuzione agli artisti», AAS 56 (1964) 438-444. 


\section{PROSPETTIVE E ALCUNE CONCLUSIONI}

Forse mai come oggi gli uomini e le donne contemporanei sperimentano l'urgente necessità di dare un senso alla propria esistenza. In una certa misura, nella loro ricerca, sono disposti ad ascoltare, sono disposti a meravigliarsi, a lasciarsi abbagliare da una parola su Dio che li porti a ri/scoprire la Parola di Dio, se questo processo è percepito come vivo e performante.

La storia dell'arte, della teologia e lo studio della liturgia, dimostrano come liturgia e teologia sono correlate alla realtà musicale: «così la Chiesa nella sua dottrina, nella sua vita e nel suo culto, perpetua e trasmette a tutte le generazioni tutto ciò che essa è, tutto ciò che essa crede» (Dei Verbum, n. 8). C’è un intenso rapporto tra l'evoluzione -nel senso dello sviluppo storico- della teologia e lavanzare delle arti e della musica naturalmente; così come anche della liturgia e la loro particolare inculturazione musicale. Ho elencato le intime relazioni tra il mondo dell'arte e della comprensione del mistero de Dio e il culto a Lui dovuto. Lunione di arte, musica e liturgia hanno dato ottimi risultati concretizzati in vere opere d'arte che rivelano il mistero. Mi chiedo se quest'unione ammirevole è stata depauperata dal nostro mondo attuale, o è solo la distrazione dell'uomo post-post-moderno, super- impegnato nel suo lavoro malaticcio, ad averla resa infruttuosa. Qui ho voluto mostrare l'intima relazione tra il mondo dell'arte visivo e uditivo e la comprensione misterica di Dio nel centro nevralgico della celebrazione liturgica e la rivelazione di Dio che porta all'assentimento della fede. Lalleanza cristianesimo-arte, Chiesa-cultura, arte-liturgia e divenuta sterile? Oppure soltanto siamo incapaci di analizzare, di ammirare o capire la grazia che si rivela in questa singolare unione?

Il Magistero della Chiesa -in particolare san Pio X, il Beato Papa Paolo VI e San Giovanni Paolo II- non ha dimenticato queste domande e abbiamo visto la grande stima che è riservata al musicale nella liturgia e al ruolo dell'arte nella predicazione del Vangelo. La stessa evoluzione del pensiero dell'uomo che si rivolge al nichilismo, alla visione del mondo come assurdo, la morte di Dio, non trova nessun punto d'incontro con l'arte liturgica. La durezza del mondo contemporaneo, la crudeltà della guerra, la nascita di un gusto globale e il pensiero comune, non lascia 
spazio a un linguaggio capace di essere considerato come un linguaggio dell'arte, al servizio della Chiesa tramite nella liturgia, il luogo per eccellenza della percezione sacramentale del Mistero.

A questo punto credo che sarebbe necessario promuovere lo studio della teologia musicale e i principi teologici nella musica; tentare di studiare la percezione del mistero e di determinare il ruolo "quasi" sacramentale della musica in questa percezione. Potrebbe essere giunto il momento di chiedersi come studiare la dinamica dell'emozione della musica e di essa nella liturgia. Ci sono nuovi tentativi di analizzare l'arte, la bellezza, come un luogo di epifania del mistero. Grandi teologi lo hanno fatto: S. Agostino ${ }^{37}$, Hans Urs von Balthasar ${ }^{38}$, Pierangelo Sequeri ${ }^{39}$, Joseph Ratzinger ${ }^{40}$. Essi hanno considerato l'esperienza della bellezza, potremmo dire l'esperienza mistica e liturgica della bellezza, come un percorso diretto per l'esperienza del mistero: un'esperienza della rivelazione di Dio che si traduce in un sensus fidei che può diventare comunione.

Oggi continua e vive una ricerca di questa bellezza che manifesta il trascendente. L'esperienza estetica è uno dei pochi modi che ancora permette agli uomini e alle donne contemporanei di avvertire il peso esperienziale del Mistero. Lestetica è una delle poche lingue che abbiamo per comunicare con gli uomini e le donne di oggi, così lontani dall'esperienza interiore e che vivono solo verso l'esterno. Rimane uno dei pochi modi per dire la meraviglia del mistero che comunica e parla attraverso il linguaggio della bellezza artistica, attraverso l'emozione dellesperienza che muove l'«affetto".

Alcuni autori hanno parlato di arte come operazione simbolica, come luogo dove si può percepire ciò che non è percepibile. La musica, in particolare, ha questa qualità. L'ineffabilità della musica, l'intangibilità dell'arte dei suoni, la fanno metafora viva del mistero. L'esperienza musicale è infatti l'unico modo per capire qualcosa di se stessa. Questa esperienza musicale è fatta nel più intimo, nella regione in cui qualcosa

37 Augustinus, Ordine, Musica, Bellezza, ed. M. Bettetini, Rusconi, Milano 1992.

${ }^{38}$ H .U. von Balthasar, «Lo sviluppo dell'idea musicale. Testimonianza per Mozart», in P. Sequeri, Antiprometeo. Il musicale nellestetica teologica di Hans Urs von Balthasar, Glossa, Milano 1995, 13-47.

39 P. Sequeri, «Il teologico e il musicale», Teologia de Milano 10 (1985) 307-338.

40 J. Ratzinger, La festa della fede, Jaca Book, Milano 1984. 
dentro l'essere umano vibra con tutti i sensi. Vibra lo stesso luogo in cui lo spirito umano ha la percezione del Mistero.

Ecco un ammirevole dialogo: la lode di Dio è santificazione degli uomini e a sua volta apre al dialogo con gli uomini e le donne che cercano, a volte senza saperlo, Dio. E qui si radica il fondamento teologico: la liturgia è fortemente implicante e la musica è un mezzo per sperimentare empaticamente il Mistero celebrato: un'implicazione che si traduce, se viva, in missione.

Se la musica è intesa come un oggetto capace di trasmettere l'esperienza del Mistero può essere intesa nel senso «tillichiano» di sacramentale ${ }^{41}$, che diventa l'indicatore del Mistero che ci dona di comprendere. Tuttavia, se fine a se stessa diventa divisione, suggestione e inganno. Il culto cristiano pone in relazione la musica e la Parola per evitare questa tentazione. Da quest'unione è nata ricca e immensamente feconda una comprensione particolare sacramentale della musica come percezione trascendentale del mistero. Quindi possiamo dire che la musica è tra i canali sacramentali che ci permette la percezione del mistero, ci permette di fare esperienza di questa comunicazione, ci permette di capire qualcosa del Mistero di Dio che è dentro di noi e si manifesta attraverso la vibrazione dellarte.

Teologi, architetti, artisti, musicisti devono dire una parola sull'Ineffabile e sul mistero della sua comunicazione, sulla stessa percezione del Mistero di Dio. Penso che nei nostri giorni non possiamo eludere, come teologi, e meno come cristiani, i meta-linguaggi. Nell'arte e nel suo excessus, nella percezione estetica che porta aldilà dei limiti delle parole, possiamo trovare il cammino di comunicazione della percezione, anche estetica, del Mistero di Dio che si rivela. Spazio, tempo e finitudine, sono gli ambiti, le categorie finite, che ci permettono di capire qualcosa della rivelazione e fanno che essa sia comprensibile per l'essere umano, finito in se stesso, ma capax Dei e capace di capire empaticamente la trascendenza del Dio che si rivela definitivamente in Gesù Cristo ${ }^{42}$.

${ }^{41}$ P. Tillich, Teologia sistematica, Claudiana, Torino 1996, 248.

${ }^{42}$ Come recita una bellissima colletta del rito Hispano-Mozarabico: «Signore nostro Dio, poni la tua mano sulla nostra testa; dacci sensi puri per vederti; dacci sensi umili per ascoltarti. Dacci sensi di amore per servirti. Dacci sensi di fede per rimanere santamente in te. Infondi, o Signore nel nostro cuore desideri che tu solo sia in grado 


\section{BIBLIOGRAFIA}

Adorno Th. W., Philosophie der neuen Musik, Mohr, Tübingen 1949.

Augustinus Aurelius, Confessionum libri XIII, ed. L. Verheijen, Corpus Christianorum Series Latina, 27, Brepols, Turnhout 1981.

Augustinus, Ordine, Musica, Bellezza, ed. M. Bettetini, Rusconi, Milano 1992.

Balthasar H.U.von, "Lo sviluppo dell'idea musicale. Testimonianza per Mozart», in P. Sequeri, Antiprometeo. Il musicale nellestetica teologica di Hans Urs von Balthasar, Glossa, Milano 1995, 13-47

Balthasar H.U. von, «Offenbarung und Schönheit», in Verbum Caro: Skizzen zur Theologie, Johannes Verlag, Einsideln 1960.

Benedetto XVI, «Litterae Apostolicae Motu Proprio datae quibus Annus Fiedi Incohatur» „Porta Fidei”, AAS 103 (2011) 723-734.

Bugnini A., La riforma liturgica 1948-1975, CLV-Edizioni Liturgiche,, Roma 1997.

Canobbio G., Pequeño diccionario de teología, Sígueme, Salamanca 1992.

Eckholt, Poetik der Kultur. Bausteine einer interkulturellen dogmatischen Methodenlehre, Herder, Freiburg 2002.

Fubini E., Estetica della musica, Il Mulino, Bologna 2003.

Hünermann P., Dogmatische Prinzipienlehre, Aschendorff Verlag, Münster 2003.

Jankëlëvitch V., La Musica e l'ineffabile, Bompiani, Milano 2001.

Langer S., Philosophy in a New Key: a study in the symbolism of reason, rite, and art, Mentor Book, The New American Library, New York 1951.

Meyer L., Emotion and Meaning in Music, University of Chicago Press, Chicago 1956.

Paolo VI, «Alocuzione agli artisti», AAS 56 (1964) 438-444.

Piqué J., «Lattimo fuggente/sfuggente: L’universo sacramentale della musica. Dalla forma estetica all'evento empatico», in Il Corpo del Logos. Pensiero estetico e teologia cristiana, ed. P. Sequeri, Glossa, Milano 2009, 179-195.

Piqué J., «Lorecchio pensante. Ascoltare il nome trinitario di Dio. Dal Gregoriano a W.A, Mozart e Ch. Gounod», in La liturgia alla prova del sacro, ed. P. Tomatis, Atti della XXXIX Settimana di Studio dell'Associazione Professori di Liturgia, Convegno Nazionale dei Professori di Liturgia Italia, Brescia 1 settembre 2011, (Studia Liturgica / Nova serie / 57), CLV-Edizioni Liturgiche, Roma 2013, 245-278.

Piqué J., «Livre du Saint Sacrement. Eucharistie als Manifestation von Erfahrung und Transzendenz», in Musik des Unsichtbaren. Der Komponist Olivier Messiaen (1908-1992) am Schnittpunkt von Theologie und Musik, ed. Michaela Christine Hastetter, EOS Verlag, St. Ottilien 2008, 104-125.

Piqué J., «Música Litúrgica: empatía y performatividad», Phase 307 (2012) 74-78.

di soddisfare; metti sulle nostre labbra preghiere che solo Tu possa ascoltare; metti nelle nostre mani opere che solo tu possa benedire. Illuminaci sempre con lo splendore della tua luce, fa' che siamo riflesso della tua Bellezza. AMEN». 
Piqué J., «Música Sacra/Música Litúrgica: Lenguaje musical y liturgia a cincuenta años de Sacrosanctum Concilium», Phase 317 (2013) 501-515.

Piqué J., Teologia e musica. Dialoghi di trascendenza, (Comunicazione/studi), San Paolo, Milano 2013.

Piqué J., «The Fleeting Moment: The Sacramental Universe of Music, from the Aesthetic Form to the Emphatic Event. Theology and Music: Languages of Transcendence», Res 7 (3/2015) 169-183.

Piqué J., Teología y música: una contribución dialéctico-trascendental sobre la sacramentalidad de la percepción estética del Misterio. Agustín, Balthasar, Sequeri; Victoria, Schönberg, Messiaen, Tesi Gregoriana, Serie Teología 132, Editrice Pontificia Università Gregoriana, Roma 2006.

Planellas J., «Prolusió (4 d’octubre de 2012). Commemoració del cinquantenari de l'inici del Concili Vaticà II», Revista Catalana de Teologia 38 (2013) 344-356.

Ratzinger J., Cantate al Signore un canto nuovo, Jaca Book, Milano 1996.

Ratzinger J., La festa della fede, Jaca Book, Milano 1984.

Sacra Congregatio Rituum, «Instructio De Musica in Sacra Liturgia. Musicam Sacram», AAS 59 (1967) 300-320.

Salmann E., Presenza di Spirito, Messaggero, Padova 2000.

Sequeri P., «Il teologico e il musicale», Teologia de Milano 10 (1985) 307-338.

Sequeri P., Antiprometeo. Il musicale nellestetica teologica di Hans Urs von Balthasar, Glossa, Milano 1995, 13-47.

Sequeri P., Musica e mistica, Librería Editrice Vaticana, Città del Vaticano 2005.

Staglianò A., Il Mistero del Dio Vivente, EDB, Bologna 1996.

Stefani G., «Musica, Chiesa, Società. A 30 anni dal Concilio Vaticano II», in La Musica e il Sacro. Atti dell'Incontro Internazionale di Studi, ed. B. Brumana - G. Giliberti, Firenze 1997, 233-241.

Tillich P., Teologia sistematica, Claudiana, Torino 1996. 